\title{
New weak-line T Tauri stars in Orion from the ROSAT all-sky survey $^{\star}$
}

\author{
J.M. Alcalá ${ }^{1,7}$, L. Terranegra ${ }^{2}$, R. Wichmann ${ }^{3}$, C. Chavarría-K. ${ }^{4}$, J. Krautter ${ }^{3}$, J.H.M.M. Schmitt ${ }^{1}$, M.A. \\ Moreno-Corral ${ }^{5}$, E. de Lara ${ }^{5}$ and R.M. Wagner ${ }^{6}$ \\ 1 Max-Planck-Institut für Extraterrestrische Physik, D-85740 Garching, Germany \\ 2 Osservatorio Astronomico di Capodimonte, Via Moiariello 16, I-80131 Napoli, Italy \\ 3 Landessternwarte-Königstuhl, D-69117 Heidelberg, Germany \\ 4 Max-Planck-Institut für Astronomie Königstuhl, D-69117 Heidelberg , Germany \\ 5 Instituto de Astronomía, Ensenada B.C., México CP 22860 \\ 6 Lowell Observatory, 1400 West Mars Hill Road. Flagstaff, Az 86001, U.S.A. \\ 7 Instituto Nacional de Astrofísica Optica y Electrónica, A.P. 51 y 216 C.P. 72000, Puebla, México
}

Received September 20, 1995; accepted January 26, 1996

\begin{abstract}
We present results of the spectroscopic and photometric follow-up observations of the ROSAT allsky survey in the direction of the Orion cloud complex. The main goal of these observations is the search for X-ray emitting pre-main sequence stars. 820 X-ray sources were detected with high confidence in about 450 square degrees. The mean density of X-ray sources in this region is a factor of about two higher than that of the whole RASS. 5\% of the RASS sources in this region are identified with previously known and likely pre-main sequence stars. We have investigated spectroscopically 181 new RASS sources widely distributed over the entire cloud complex. On the basis of the presence of strong Li I $\lambda 6707$ absorption, spectral type later than F0 and chromospheric emission, 112 new weak-line T Tauri stars could be found. We present coordinates, X-ray count-rates and finding charts of the new PMS. Optical $U B V(R I)_{\mathrm{KC}}$, near-infrared $J H K L M$ and $u v b y$ - $\beta$ photometry for the new WTTS is also provided. In addition $24 \mathrm{dKe}-\mathrm{dMe}$ stars were also found on the basis of the RASS data.
\end{abstract}

Key words: X-rays: stars — stars: pre-main-sequence — ISM: Orion star forming region

\section{Introduction}

From observations carried out with the EINSTEIN satellite in star forming regions (SFRs), it is well known that $\mathrm{T}$ Tauri stars can be detected in X-rays with luminosities in the range $L_{\mathrm{X}}=10^{29}-10^{32} \mathrm{erg} / \mathrm{s}$ (Feigelson 1987; $\mathrm{Ku} \&$ Chanan 1979; Walter et al. 1988, and references therein). These observations led to the discovery of a large number of "weak-line" T Tauri stars (WTTS). These lowmass $\left(M \leq 3 M_{\odot}\right)$ pre-main sequence (PMS) stars as their classical counterparts, the classical T Tauri stars (CTTS), show strong Li I $\lambda 6707$ absorption in their optical spectra. Since Li I is rapidly destroyed in the convective layers of low-mass stars in the early phases of the stellar evolution (Bodenheimer 1965), its presence is used as an indicator of extreme youth. While the CTTS can be easily detected

Send offprint requests to: J.M. Alcalá

${ }^{\star}$ Partially based on observations collected at the German Spanish Astronomical Center in Calar Alto, Spain, at Observatory of the Ohio State University in Flagstaff, Arizona, U.S.A. and at the San Pedro Mártir Observatory, UNAM, México by their strong Balmer emission lines in objective-prism surveys or through their IR excesses, WTTS lack both characteristics. This has been ascribed to the absence of dense circumstellar matter in WTTS. Thus, WTTS are more easily detected on the basis of their X-ray emission.

Before the survey carried out by the X-ray satellite ROSAT (Röntgensatellit), all X-ray observations in SFRs were strongly concentrated towards specific areas containing CTTS, and no information about the spatial distribution of X-ray sources far from molecular clouds was available. The ROSAT all-sky survey (RASS) provides a spatially unbiased sample of X-ray sources at about the same sensitivity of EINSTEIN pointed mode observations thus offering the possibility to carry out an extensive study of the spatial distribution of X-ray emitting PMS stars by using a spatially complete sample of X-ray sources.

With the aim to identify the RASS X-ray sources in SFRs, a long-term project was started (Krautter et al. 1994). The 
main purpose of this program is the search for WTTS in nearby (closer than $500 \mathrm{pc}$ ) SFRs.

The Orion cloud complex at a distance of about 460 pc (Genzel \& Stutzki 1989), is a suitable region to study the star formation process, the content of the young stellar population and the interaction of the stellar members with their environment. X-rays observations in Orion have been carried out for the first time by the Uhuru satellite (Giacconi \& Gursky 1974) and by the EINSTEIN satellite (Gagné \& Caillault 1994, and references therein). However, all these observations have been restricted to regions in or near the Orion nebula, with no information about the possible presence of X-ray emitting PMS stars far from the cloud complex.

In this paper we report the observations of the RASS in the direction of the Orion SFR and focus on the optical identification of the detected X-ray sources. We give coordinates, X-ray count rates and finding charts of the Xray sources identified spectroscopically as new WTTS. We also provide $U B V(R I)_{\mathrm{KC}}, J H K L M$ and $u v b y$ - $\beta$ photometry for a sub-sample of the new WTTS. In a forthcoming paper we analyse the optical counterparts identified as low-mass PMS stars, in particular, relationships between their location in the cloud and the main stellar parameters, and of the latter with X-ray properties of the studied stars.

We stress that very large samples of X-ray sources, like the RASS sample in Orion, require several years of identification work. Excluding the sources previously known from EINSTEIN observations in this SFR, the sample of RASS sources identified in our spectroscopic study is complete to about $20 \%$ by now. Since the RASS is flux limited and because of the large distance of the Orion SFR, this sample may be biased towards the brightest X-ray sources. Although we knew in advance that such a program of spectroscopic identifications would require several years to be completed, we think it is very important to publish the sample of new WTTS. Such a sample is very suitable for future studies of PMS stellar activity and its connection with the evolution of angular momentum in the early phases of the stellar evolution by measuring rotational velocities through high-resolution spectroscopic observations and/or photometric determinations of rotational modulations.

\section{The ROSAT all-sky survey in Orion}

The telescope, the position-sensitive proportional counter (PSPC) detector and the observing procedure used during the RASS are discussed in detail by Trümper et al. (1991) and Pfeffermann et al. (1986). RASS exposure times depend on the ecliptic latitude $\beta$. For the Orion SFR, the exposure times range from $350 \mathrm{~s}$ to $800 \mathrm{~s}$.

From previous recent studies of RASS X-ray sources in nearby SFRs, it is known that WTTS occupy a larger area on the sky than the CTTS (Alcalá et al. 1995, hence- forth A95; Wichmann et al. 1995, henceforth W95). For our study in Orion, we have selected an area within the (J2000) coordinates: $\alpha_{\min }=5^{\mathrm{h}}, \alpha_{\max }=6^{\mathrm{h}}, \delta_{\min }=-14^{\circ}$, $\delta_{\max }=+16^{\circ}$. This area includes Orion's bright nebula, the molecular clouds A (L1641) and B (L1630) described by Maddalena et al. (1986) and Blaauw (1991). The $\lambda$ Orionis region located at the north of the Orion OB1 association is also included. Within the selected area, 820 X-ray sources were detected with high confidence using the processing of the Standard Analysis Software System (SASS) (Voges et al. 1992a).

Of particular interest is the density of X-ray sources found in this region. While the mean density of sources for the whole RASS is about 1 , there are about 2 sources per square degree in the Orion SFR.

\subsection{Source detection}

The processing of the RASS data was performed using the Extended Scientific Analysis System (EXSAS, Zimmermann et al. 1994) as described in A95 and Neuhäuser et al. (1995). From this processing, the X-ray count rate for each source, the corresponding count errors and the likelihood of the detection are obtained. The typical $(2 \sigma)$ X-ray error circle for most of the sources has a radius not larger than $40^{\prime \prime}$, which is in good agreement with the determination of Neuhäuser et al. (1995) for RASS sources in the Taurus-Auriga region. All the SASS X-ray sources were recovered in the EXSAS processing. Thus, the identification described below was performed on the 820 X-ray sources detected from both the SASS and the EXSAS processing. For the search of optical counterparts we used a $40^{\prime \prime}$ radius as a high confidence level error circle.

The spatial distribution of the RASS X-ray sources in the Orion SFR has been discussed by Sterzik et al. (1995). They used 98 of the 112 new WTTS found in the present work (see Sect. 3.2) as the WTTS training sample to preselect potential PMS candidates. In their predicted spatial distribution they find the presence of different concentrations of X-ray emitting PMS stars rather far from the star forming dark clouds.

\subsection{Variable $X$-ray sources}

Following the procedure described in A95, we obtained X-ray light curves for some 200 RASS sources detected with a count rate greater than $0.08 \mathrm{cts} / \mathrm{s}$. Only the X-ray sources RXJ0534.0-0221, RXJ0530.3-1308 and RXJ0534.6+1007 show significant variations (Fig. 1). The source RXJ0534.6+1007 is identified with a new WTTS (see Sect. 3.2 and Table 3), while the sources RXJ0534.00221 and RXJ0530.3-1308 are identified with previously unknown active stars, the former with a dM1e and the latter with a dK2e (see also Sect. 3.2). Other sources may have apparently small scale variability. However, the low signal-to-noise of the RASS data prevent us on carrying 
out a finer analysis of the light curves and to determine the nature of this variations. Thus, we cannot exclude the presence of low-level variability observable as scan-to-scan variations.

\section{Identification of the X-ray sources}

The identification of the X-ray sources consisted of two steps: first, all positional coincidences with objects contained in several astronomical catalogues held in the SIMBAD database and other catalogues (see below) were searched; secondly, the new X-ray sources were investigated by means of mid-resolution spectroscopic observations. Since our main goal in this investigation is the search for X-ray emitting PMS stars, only those sources for which a likely PMS counterpart was found in the present work or elsewhere will be considered hereafter.

\subsection{Identification in catalogues}

In Table 1 we list the coordinates and count rates of the RASS sources in Orion identified with previously known and likely PMS stars. In Col. (8) of this table we list the (broad-band X-ray flux) count rates, while in Col. (10) we give the distance between the X-ray position and it optical counterpart. These identifications were carried out by positional cross correlation with the SIMBAD catalogue, the Herbig \& Bell catalogue (1988) and the catalogues $\mathrm{H} \alpha$ line emission objects and/or variables associated with the Orion SFR by Duerr et al. (1982) which is a list of $\mathrm{H} \alpha$ emission line objects found on the basis of an objectiveprism survey in the $\lambda$ Orionis region and the catalogue by Brand \& Wouterloot (1990) which compiles a list of about 1385 variable and emission line stars in the Orion SFR. An optical PMS counterpart was associated to a RASS X-ray source when their respective positions were coincident within the $2 \sigma$ error circle given by ROSAT. The source RXJ 0534.7-0524, identified with HBC447, was included in our spectroscopic and photometric observations described below in order to confirm it PMS nature.

Forty-six RASS X-ray sources could be identified with very likely PMS stars. Most of these stars have been discovered on the basis of their strong photometric variability or because of their emission line spectra taken in photographic plates or films with an objective-prism Schmidt camera. Thus, most of their optical counterparts are expected to be CTTS. However, this still represents a rather small fraction $(5.6 \%)$ of the X-ray emitting PMS population discovered with ROSAT, i.e. the WTTS (see Sect. $3.3)$.

\subsection{Spectroscopic identifications}

The spectroscopic observations were carried out using Boller and Chivens Cassegrain spectrographs coupled with the $1.8 \mathrm{~m}$ telescope of the Ohio State University (OSU)
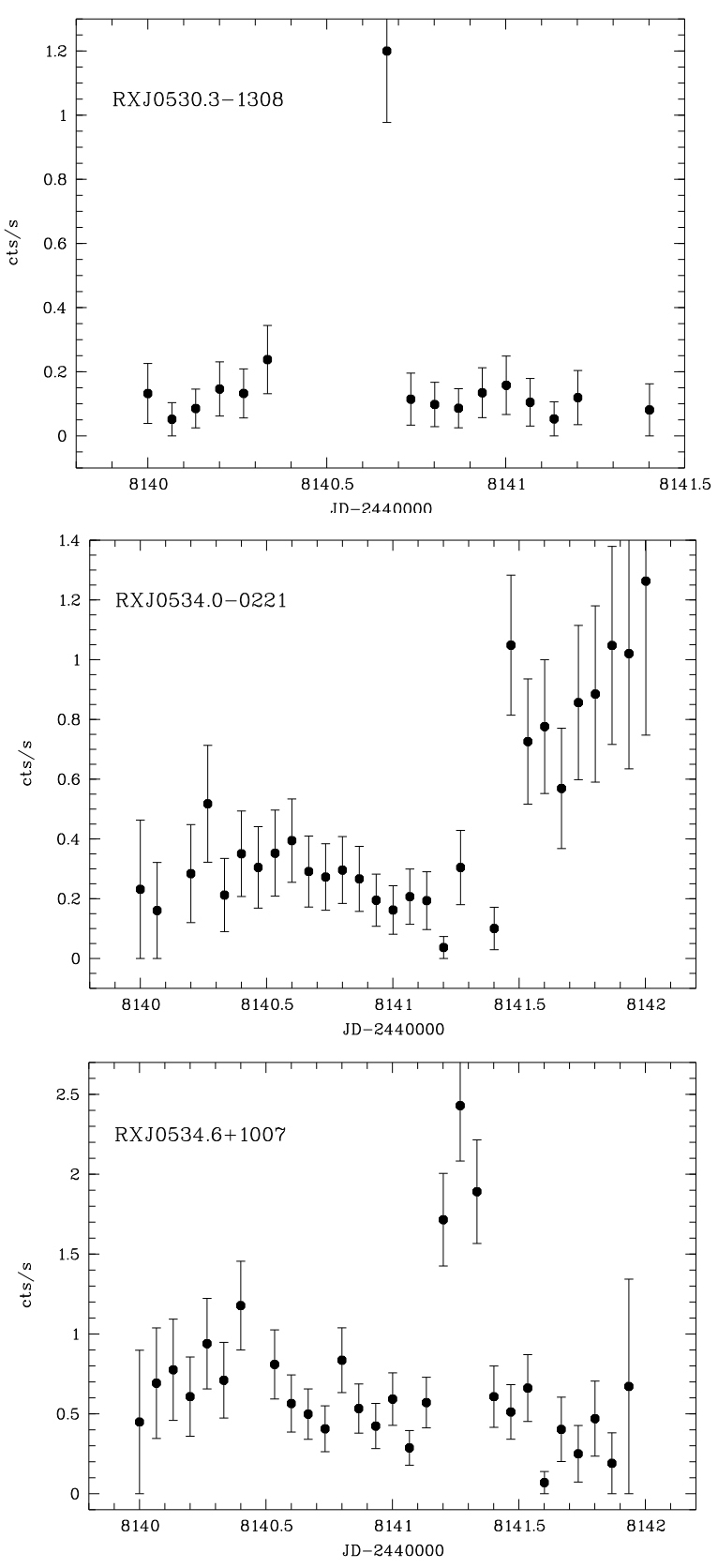

Fig. 1. X-ray light curves of the RASS sources RX 0534.00221, RXJ 0530.3-1308 and RXJ 0534.6+1007

at the Lowell Observatory in Flagstaff, Arizona, and with the $2.2 \mathrm{~m}$ telescope of the Max-Planck Institut für Astronomie at the Calar Alto (CAO) Observatory in Spain. In Table 2 we give information on the observing runs and the instrumental set-up. The spectral resolution given in Table 2 was estimated from the FWHM of the lines on the comparison spectra.

For the observations we produced finding charts using the HST catalogue database in the form of digitized photographic plates and a program available at the MPE in Garching (Voges 1992a, b). More informations are 
Table 1. X-ray sources identified with previously known and likely PMS stars

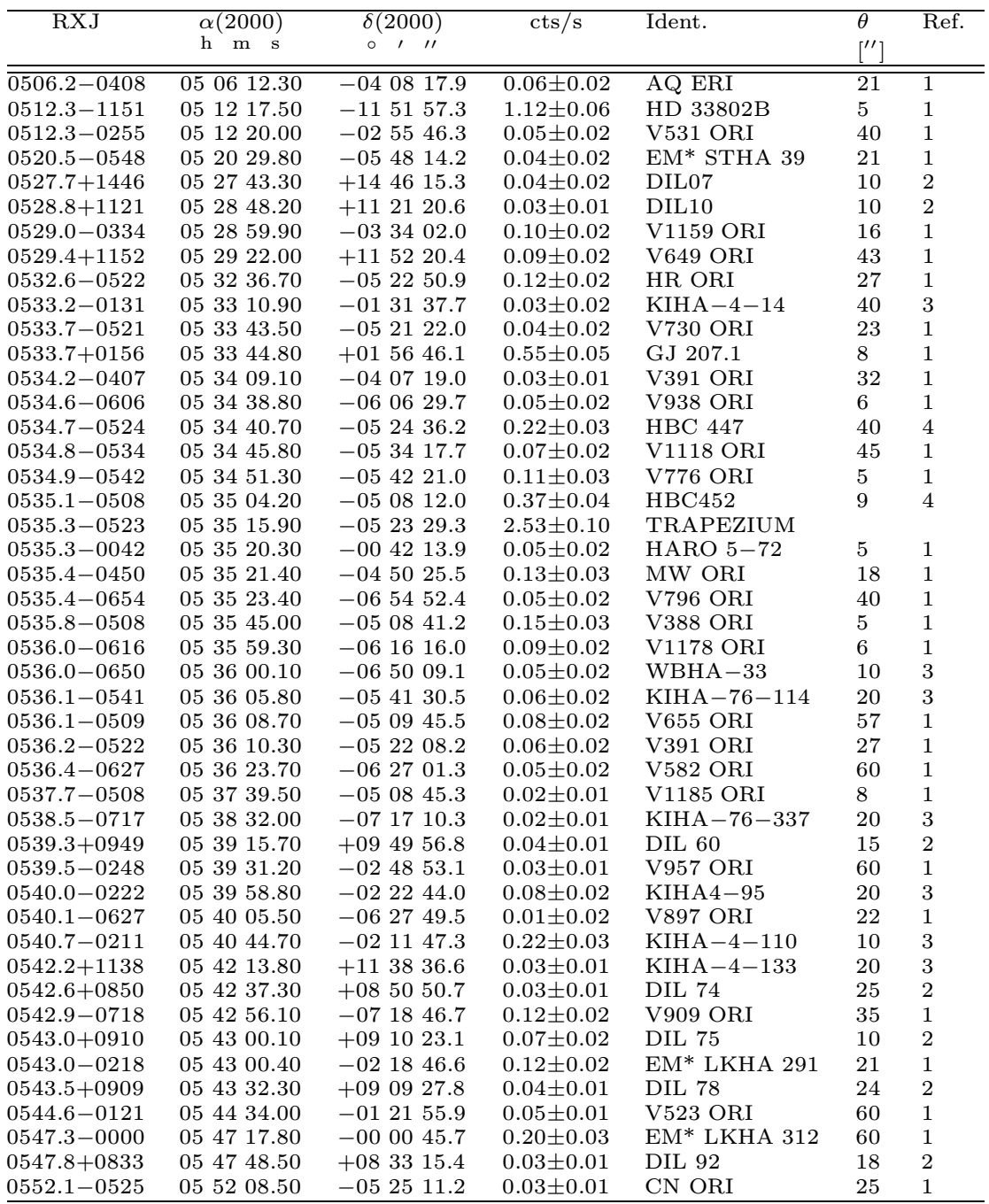

(1) Simbad catalogue ; (2) Duerr et al. (1982); (3) Brand \& Wouterloot (1990); (4) Herbig \& Bell (1988).

Table 2. Journal of spectroscopic observations

\begin{tabular}{lllccccc}
\hline Tel. & Period & CCD & $\begin{array}{c}\text { Disp. } \\
{[\AA / \mathrm{mm}]}\end{array}$ & $\begin{array}{c}\text { Range } \\
{[\AA]}\end{array}$ & $\begin{array}{c}\text { Res. } \\
{[\AA]}\end{array}$ & $\begin{array}{c}\text { No. of Sources } \\
\text { investigated }\end{array}$ & $\begin{array}{c}\text { No. of new } \\
\text { WTTS }\end{array}$ \\
\hline OSU1.8m & $20.12 .91-27.12 .91$ & TI 4849 & 150 & $4500-7000$ & 11.5 & 65 & 38 \\
CAO2.2m & $28.12 .91-02.01 .92$ & RCA\#11 & 72 & $5500-6800$ & 3.9 & 24 & 18 \\
CAO2.2m & $22.12 .92-29.12 .92$ & TEK\#6 & 72 & $5500-6800$ & 4.0 & 29 & 16 \\
OSU1.8m & $02.12 .93-09.12 .93$ & TEK & 72 & $5500-6800$ & 5.0 & 28 & 21 \\
CAO2.2m & $16.12 .93-26.12 .93$ & TEK\#6 & 72 & $5500-6800$ & 3.7 & 35 & 19 \\
\hline
\end{tabular}

obtained from the output of this program apart of the finding chart itself. Namely, the X-ray error circle is drawn and a list of coordinates and photographic magnitudes, $B_{\mathrm{ph}}$, of different types of objects (with the specification if star, galaxy or faint), sorted by increasing distance from the $\mathrm{X}$-ray source position, is provided.
In order to avoid any bias in the spatial distribution, we divided the studied area in 12 regions (6 in the coordinate range $5^{\mathrm{h}} \leq \alpha \leq 5^{\mathrm{h}}: 30^{\mathrm{m}}$ with $\delta$ running form $-14^{\circ}$ to $+16^{\circ}$ in intervals of $5^{\circ}$, and the other 6 regions in the coordinate range $5^{\mathrm{H}}: 30^{\mathrm{m}} \leq \alpha \leq 6^{\mathrm{h}}$ with $\delta$ running in the same way). The number of investigated $\mathrm{X}$-ray sources is approximately the same $(\approx 15 \pm 1)$ in each region. Thus, 
the sample of X-ray sources investigated spectroscopically is spatially unbiased.

For each field observational priority was assigned mainly on the basis of the brightness of the objects and their relative position to the error circle: highest priority was given to stars inside the error circle and brighter than about $B_{\mathrm{ph}}=17$. From Einstein observations in SFRs and from the $f_{x} / f_{v}$ ratio for known $\mathrm{T}$ Tauri stars, it is expected that X-ray emitting T Tauri stars have $V$ magnitudes in the range 10-16. Furthermore, the T Tauri stars identified in catalogues (Sect. 3.1) are normally brighter than $V=15$. Since typical $B-V$ colours of T Tauri stars are in the range of $0.3-1.6$, we adopted $B_{\mathrm{ph}}=17$ as a reasonable limiting magnitude in the search for WTTS (which was also the limiting magnitude of our set-up to get a good sinal-to-noise ratio in reasonable exposure times). If no star brighter than about $B_{\mathrm{ph}}=17$ was inside the error circle, the stellar object nearest to the X-ray PSPC position (within a radius of about $60^{\prime \prime}$ ) and brighter than about $B_{\mathrm{ph}}=17$ was observed. Sometimes more than one star brighter than $B_{\mathrm{ph}}=17$ was found inside the error circle. In such cases, all these stars were observed.

Typically, 2-3 candidates were observed in each X-ray error circle. The exposure times for the spectroscopic observations were in the range 10-15 minutes for the brightest stars $\left(V \leq 13^{\mathrm{m}}\right)$, and up to $20-35$ minutes for the faintest ones $\left(V \leq 16^{\mathrm{m}}\right)$. With these exposure times, a $S / N \approx 70$ was achieved in the best cases. For the wavelength calibration of the stellar spectra, a He-Ar lamp was used to obtain comparison spectra immediately after each object exposure. For flux calibration purposes, 2 to 3 standard stars per night were also observed nightly.

In order to carry out the spectral type classification, a grid of bright $\left(3^{\mathrm{m}} \leq V \leq 6^{\mathrm{m}}\right)$ standard stars with very well determined spectral types was observed using the same instrumental set-up.

All the spectra were reduced following the standard procedure of MIDAS software package. A relative flux calibration of each spectrum was performed using a mean response function for each observational run. Since most of the nights were not photometric, only a relative flux calibration of the spectra could be performed.

To classify a star as a WTTS we adopted the same criteria as in A95, namely: presence of the Li I $\lambda 6707$ absorption line, spectral type later than F0 and eventually the presence of chromospheric $\mathrm{H} \alpha$ emission with equivalent width less than $10 \AA$. However, the latter is not necessarily a PMS indicator (Walter et al. 1988), since chromospherically active dMe stars show rather strong emission lines like $\mathrm{H} \alpha$ and $\mathrm{Ca}$ II $\mathrm{H}+\mathrm{K}$, but they are not PMS stars. The $\mathrm{K}$ and $\mathrm{M}$ stars with $\mathrm{H} \alpha$ in emission but lacking Li I absorption have been classified by us as dKe and dMe stars, respectively.

181 spatially unbiased RASS X-ray sources were investigated spectroscopically in Orion. Of these, 110 were found to be associated with 112 stars showing PMS characteristics (two of these identifications are ambiguous, see below). Other 9 sources are identified with stars showing clear $\mathrm{H} \alpha$ emission or filled-in with emission and marginal evidence of lithium in absorption, but need confirmation because of the low signal-to-noise of their spectra. In addition, 24 sources were found to be associated with previously unknown dKe-dMe stars unrelated to the SFR. Some of these active stars display clear emission at $\mathrm{H} \alpha$ but others show some emission filling-in the line. For the rest of the sources, no stellar counterpart with $V \leq 17^{\mathrm{m}}$, i.e. fainter than the limiting magnitude of our spectroscopic observations, could be found. By inspecting the finding charts, we find that the error circles of a major part of these sources contain extragalactic objects and/or faint $\left(V \geq 18^{\mathrm{m}}\right)$ stellar-like objects. Thus, very likely the optical counterparts of these sources are extragalactic objects.

In total, some 438 spectra were obtained. About $60 \%$ of the 181 investigated X-ray sources resulted in positive WTTS identifications. This number is fully consistent with the detection rate of RASS discovered WTTS in the Chamaeleon and Taurus-Auriga SFRs (A95, W95). The coordinates and the (broad X-ray flux) count rates of the X-ray sources identified with new WTTS are given in Table 3. In this table, we also include the 9 WTTS candidates for which the presence of lithium absorption has to be confirmed. In Fig. 2 typical spectra of the new WTTS in Orion are shown.

A few sources have ambiguous optical identification, since more than one new PMS star is found in the same error circle. Two sources (RXJ0532.4+0131 and RXJ0538.4-0637) have two WTTS in the same error circle. Also, a WTTS and a dKe star were fount to be associated to the source RXJ0532.4-0713 and two sources (RXJ0500.9+0222 and RXJ0549.7-1158) have two dKedMe stars in the same error circle.

The total number of CTTS and WTTS in Orion is still very uncertain. The spectroscopic identification of the RASS sources is far from being complete, and there are many emission line objects in this region for which the PMS nature is not yet confirmed. However, already the RASS WTTS in Orion outnumber the stars in Table 1 by a factor of about 2.5 .

\subsection{Spectral types and equivalent widths}

Spectral types were determined by comparison with a grid of bright spectral standard stars (from F0 to M5) observed with the same dispersion and the same instrumental setup in each observing run, using the same procedure as described in A95. The accuracy of the spectral types is estimated to be about \pm 1 sub-class in most cases. The spectral types of the WTTS are listed in Table 3.

The equivalent widths of the $\mathrm{H} \alpha$ and $\mathrm{Li}$ I lines were obtained using the same procedure as described in A95. The mean standard deviations resulting from several 


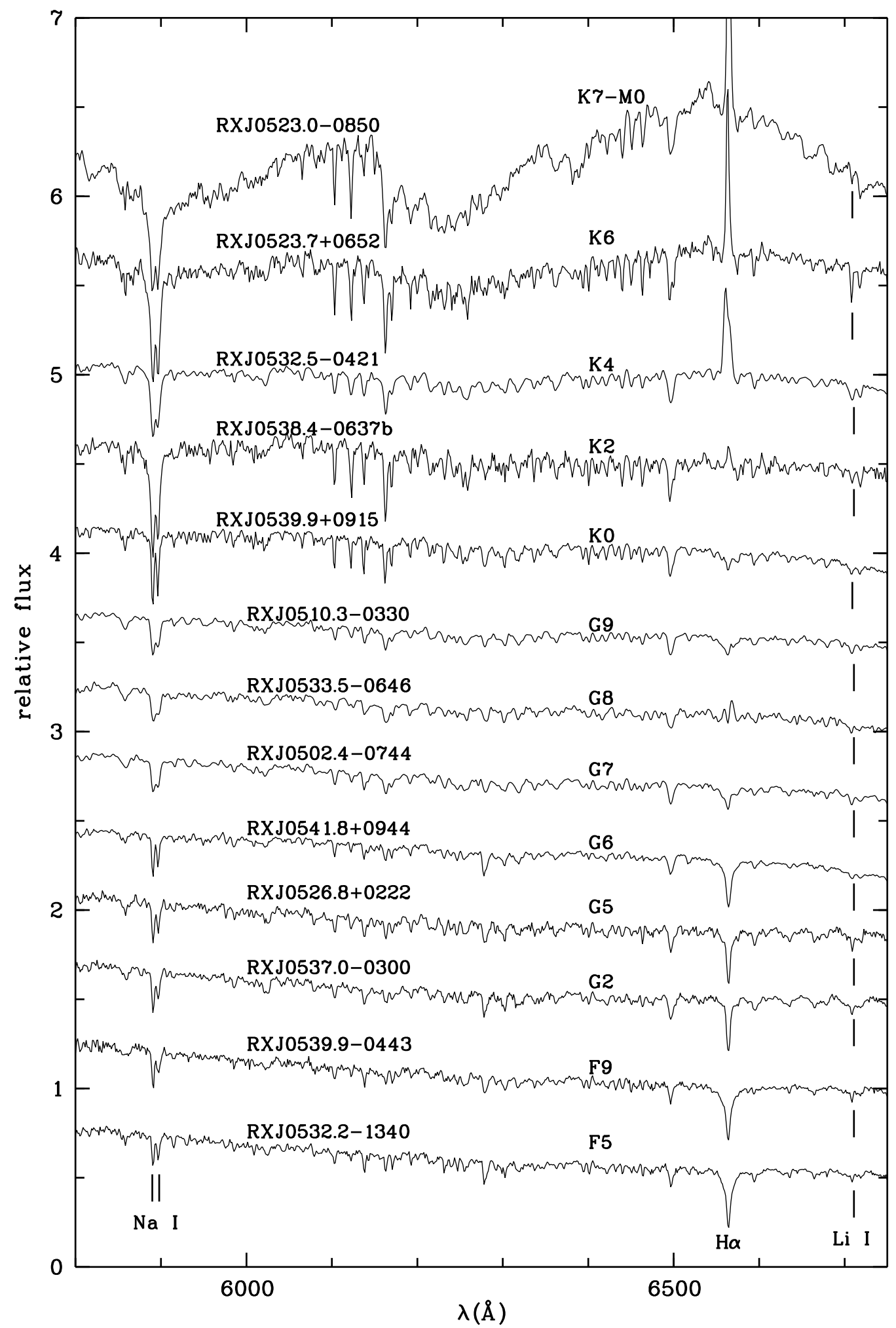

Fig. 2. Examples of typical WTTS spectra in Orion. Each spectrum is normalized to the continuum at $\lambda=6000 \AA$ and arbitrary shifted for the sake of comparison 
Table 3. RASS sources identified with new WTTS in Orion

\begin{tabular}{|c|c|c|c|c|c|c|}
\hline$\overline{\mathrm{RXJ}}$ & $\begin{array}{c}\alpha(2000) \\
\mathrm{h} \quad \mathrm{m} \\
\quad(2) \\
\end{array}$ & $\begin{array}{c}\delta(2000) \\
\circ \quad, \quad \prime \\
(3) \\
\end{array}$ & Sp.T. & $\begin{array}{r}W(\mathrm{H} \alpha) \\
{[\AA]} \\
(5) \\
\end{array}$ & $\begin{array}{c}W(\mathrm{Li}) \\
{[\AA]} \\
(6) \\
\end{array}$ & $\mathrm{cts} / \mathrm{s}$ \\
\hline $0500.4-1054$ & $\begin{array}{lll}05 & 00 & 26.7\end{array}$ & -105447.9 & K6 & -1.80 & 0.30 & $0.049 \pm 0.018$ \\
\hline $0501.1+0642$ & $\begin{array}{lll}05 & 0105.8\end{array}$ & +064203.0 & K5 & -0.55 & 0.42 & $0.043 \pm 0.013$ \\
\hline $0502.4-0744$ & $\begin{array}{lll}05 & 02 & 21.1\end{array}$ & -074402.9 & G7 & +1.70 & 0.30 & $0.050 \pm 0.015$ \\
\hline $0503.8-1130$ & 050349.5 & -113059.0 & K3 & +1.50 & 0.45 & $0.057 \pm 0.016$ \\
\hline $0506.2+0439$ & $\begin{array}{lll}05 & 06 & 13.2\end{array}$ & +043947.4 & M3 & -6.50 & 0.27 & $0.189 \pm 0.028$ \\
\hline $0507.5+1010$ & $\begin{array}{llll}05 & 07 & 31.2\end{array}$ & +101047.6 & $\mathrm{~K} 2$ & +2.30 & 0.40 & $0.103 \pm 0.026$ \\
\hline $0507.8-0931$ & $\begin{array}{lll}05 & 07 & 48.0\end{array}$ & -093144.3 & $\mathrm{~K} 2$ & +0.80 & 0.42 & $0.045 \pm 0.015$ \\
\hline $0509.0-0315$ & 050859.5 & $\begin{array}{lll}-03 & 15 & 10.3\end{array}$ & K1 & +1.80 & 0.33 & $0.060 \pm 0.016$ \\
\hline $0510.1-0427$ & $\begin{array}{lll}05 & 10 & 04.8\end{array}$ & -042754.2 & $\mathrm{~K} 4$ & -0.20 & 0.25 & $0.137 \pm 0.031$ \\
\hline $0510.3-0330$ & 051015.9 & -033000.4 & G9 & +1.40 & 0.36 & $0.059 \pm 0.023$ \\
\hline $0511.7-0348$ & 051139.5 & -034849.8 & K3 & +1.85 & 0.38 & $0.029 \pm 0.017$ \\
\hline $0512.3-0255$ & 051220.0 & -025546.3 & K3 & -6.70 & 0.45 & $0.045 \pm 0.016$ \\
\hline $0513.1+0851$ & $\begin{array}{lll}05 & 13 & 05.9\end{array}$ & +085119.6 & K3 & -19.50 & 0.25 & $0.037 \pm 0.015$ \\
\hline $0513.4-1244$ & 051322.0 & -124450.4 & G6 & +3.00 & 0.30 & $0.112 \pm 0.029$ \\
\hline $0515.6-0930$ & 051536.3 & -093049.5 & G5 & +2.50 & 0.25 & $0.207 \pm 0.036$ \\
\hline $0517.9-0708$ & 051754.9 & -070824.4 & K3 & +2.10 & 0.35 & $0.071 \pm 0.025$ \\
\hline $0518.0-1146$ & $\begin{array}{llll}05 & 17 & 57.2\end{array}$ & -114607.8 & K3 & -0.01 & 0.40 & $0.084 \pm 0.023$ \\
\hline $0518.0+0712$ & 051801.5 & +071228.0 & K3 & +0.30 & 0.57 & $0.034 \pm 0.016$ \\
\hline $0518.3+0829$ & 051820.0 & +082918.0 & G9 & +1.50 & 0.45 & $0.065 \pm 0.019$ \\
\hline $0518.6+0959$ & $\begin{array}{lll}05 & 18 & 37.8\end{array}$ & +095948.9 & $\mathrm{K} 2$ & +1.70 & 0.45 & $0.023 \pm 0.012$ \\
\hline $0519.9+0552$ & 051951.1 & +055210.7 & K6 & -1.25 & 0.58 & $0.059 \pm 0.019$ \\
\hline $0520.0+0612$ & $\begin{array}{lll}05 & 200.3\end{array}$ & +061257.0 & K3 & -0.20 & 0.60 & $0.085 \pm 0.023$ \\
\hline $0520.5+0616$ & $05 \quad 2032.4$ & +061612.9 & K3 & -0.90 & 0.50 & $0.123 \pm 0.028$ \\
\hline $0520.9-0452$ & 052055.9 & -045242.4 & F5 & +5.00 & 0.22 & $0.028 \pm 0.014$ \\
\hline $0522.1-0844$ & 052203.3 & -084418.5 & K1 & +1.45 & 0.40 & $0.035 \pm 0.015$ \\
\hline $0522.7+0014^{\dagger}$ & $\begin{array}{lll}05 & 22 & 43.8\end{array}$ & +001432.2 & M2 & -4.85 & - & $0.078 \pm 0.024$ \\
\hline $0522.8-1144^{\dagger}$ & 052247.6 & -114434.9 & M1 & -4.90 & - & $0.027 \pm 0.012$ \\
\hline $0522.9+0857$ & 052254.8 & +085750.5 & K1 & -1.05 & 0.20 & $0.261 \pm 0.042$ \\
\hline $0523.0-0850$ & 052257.5 & -085020.1 & K7-M0 & -5.43 & 0.37 & $0.072 \pm 0.021$ \\
\hline $0523.1-0440$ & 052303.2 & -044036.7 & K5 & -11.60 & 0.50 & $0.026 \pm 0.016$ \\
\hline $0523.7+0652$ & 052342.6 & +065201.2 & K6 & -4.40 & 0.60 & $0.055 \pm 0.021$ \\
\hline $0524.1+0730$ & 052406.4 & +073056.0 & K4 & -0.30 & 0.50 & $0.026 \pm 0.014$ \\
\hline $0525.3+0208$ & 052515.8 & +020856.0 & M4 & -10.00 & 0.45 & $0.056 \pm 0.019$ \\
\hline $0526.5+1510$ & 052632.5 & +151002.4 & G5 & +3.86 & 0.20 & $0.019 \pm 0.010$ \\
\hline $0526.7+0143$ & 052642.9 & +014348.7 & Ko & +0.20 & 0.50 & $0.041 \pm 0.016$ \\
\hline $0526.8+0222$ & 052647.8 & +022202.4 & G5 & +2.80 & 0.27 & $0.070 \pm 0.020$ \\
\hline $0527.7+0153^{\dagger}$ & 052741.3 & +015330.3 & K7 & -1.45 & - & $0.032 \pm 0.015$ \\
\hline $0528.0-0053$ & 052759.3 & -005319.3 & Ko & +0.25 & 0.30 & $0.028 \pm 0.014$ \\
\hline $0528.3+0326$ & $\begin{array}{lll}05 & 28 & 16.7\end{array}$ & +032639.1 & K0 & +0.50 & 0.40 & $0.044 \pm 0.016$ \\
\hline $0528.8+0048$ & 052847.3 & +004831.4 & K0 & +0.40 & 0.28 & $0.032 \pm 0.014$ \\
\hline $0528.8+0105$ & 052846.8 & +010539.6 & K4 & +0.30 & 0.35 & $0.050 \pm 0.015$ \\
\hline $0529.2-0615$ & 052913.7 & -061504.9 & K1 & +1.10 & 0.20 & $0.049 \pm 0.018$ \\
\hline $0529.4+0041$ & 052922.5 & +004109.1 & K2 & -0.01 & - & $0.048 \pm 0.017$ \\
\hline $0530.1+0041$ & 053005.3 & +004118.3 & $\mathrm{G} 2$ & +2.80 & 0.30 & $0.193 \pm 0.030$ \\
\hline $0530.7-0434$ & 053043.3 & -043459.3 & K3 & -0.04 & 0.53 & $0.050 \pm 0.018$ \\
\hline $0530.9+1015$ & 053055.5 & +101505.6 & K3 & +0.80 & 0.52 & $0.067 \pm 0.019$ \\
\hline $0531.2+0118$ & 053109.6 & +011852.3 & Ko & +0.45 & 0.38 & $0.022 \pm 0.012$ \\
\hline $0531.6-0326$ & 053138.6 & -032655.9 & Ko & +1.50 & 0.20 & $0.083 \pm 0.030$ \\
\hline $0532.1-0732$ & 053205.8 & -073243.9 & K4 & -0.01 & 0.31 & $0.050 \pm 0.015$ \\
\hline $0532.2-1340$ & 053214.5 & -134058.3 & F5 & +4.10 & 0.20 & $0.082 \pm 0.023$ \\
\hline
\end{tabular}

measurements of the equivalent widths are: $\sigma=0.25 \AA$ for $\mathrm{H} \alpha$ and $\sigma=0.15 \AA$ for the Li I line. For late type stars (K and $\mathrm{M}$ stars) and for stars with a low $S / N$ ratio, the uncertainty comes mainly from the fit to the continuum. The equivalent width was also measured for the stars with $\mathrm{H} \alpha$ in absorption. These stars have $\mathrm{H} \alpha$ absorption weaker than normal stars of the same spectral type. The equivalent widths are listed in Table 3 . In this table, a negative equivalent width means an emission line.

The source RXJ 0534.7-0524 is identified with the star HBC447 (see Table 1). Since its PMS nature was dubious, we decided to include this star in our spectroscopic and photometric observations. The spectrum of the star shows a double-lined peak at $\mathrm{H} \alpha$ and strong $\mathrm{Li}$ I $\lambda 6707$ $\AA$ absorption. We classified the star as a new WTTS. The spectral type we derived is in very good agreement with that given by Herbig \& Bell (1988).

\subsection{CTTS candidates?}

The dividing line between WTTS and CTTS is somewhat controversial. A parameter used to classify low-mass PMS stars as WTTS or CTTS is the equivalent width of the $\mathrm{H} \alpha$ emission line. The limiting value of $W(\mathrm{H} \alpha)=-10$ $\AA$ has been generally adopted (negative equivalent widths indicate emission lines in our convention). Nevertheless, this criterion is purely empirical and may lead to 
Table 3. continued

\begin{tabular}{|c|c|c|c|c|c|c|}
\hline RXJ & $\begin{array}{c}\alpha(2000) \\
\mathrm{h} \quad \mathrm{m} \quad \mathrm{s} \\
(2) \\
\end{array}$ & $\begin{array}{c}\delta(2000) \\
\circ \quad, \quad \prime \prime \\
(3) \\
\end{array}$ & Sp.T. & $\begin{array}{r}W(\mathrm{H} \alpha) \\
{[\AA]} \\
(5) \\
\end{array}$ & $\begin{array}{c}W(\mathrm{Li}) \\
{[\AA]} \\
(6)\end{array}$ & $\mathrm{cts} / \mathrm{s}$ \\
\hline $0532.4+0131 \mathrm{a}$ & 053222.6 & +013141.6 & K2 & +1.50 & 0.40 & $0.025 \pm 0.013$ \\
\hline $0532.4+0131 b$ & 053222.6 & +013141.6 & K5 & -0.60 & 0.60 & $0.025 \pm 0.013$ \\
\hline $0532.4-0713$ & 053224.6 & -071312.5 & K3 & -1.45 & 0.38 & $0.110 \pm 0.032$ \\
\hline $0532.5-0421$ & 053231.7 & -042140.8 & K4 & -3.50 & 0.48 & $0.032 \pm 0.014$ \\
\hline $0532.6-0522$ & $\begin{array}{lll}05 & 32 & 36.7\end{array}$ & -052250.9 & K3 & -4.00 & 0.47 & $0.120 \pm 0.024$ \\
\hline $0533.1-0758$ & 053305.0 & -075852.6 & K3 & -0.30 & 0.35 & $0.082 \pm 0.028$ \\
\hline $0533.1+0224$ & 053308.1 & +02 2456.8 & K4 & -0.20 & 0.44 & $0.038 \pm 0.015$ \\
\hline $0533.5-0646$ & 053330.5 & -064600.5 & G8 & -0.35 & 0.31 & $0.035 \pm 0.015$ \\
\hline $0534.6+1007$ & 053434.9 & +100712.8 & K3 & -0.25 & 0.36 & $0.735 \pm 0.056$ \\
\hline $0534.7-0423$ & 053440.5 & -042335.0 & $\mathrm{~K} 2$ & -0.31 & 0.29 & $0.050 \pm 0.015$ \\
\hline $\mathrm{HBC} 447^{\ddagger}$ & 053440.7 & -052436.2 & K3 & -1.80 & 0.35 & $0.219 \pm 0.034$ \\
\hline $0534.7+1114$ & 053444.3 & +11 1439.2 & $\mathrm{~K} 4$ & +0.90 & 0.32 & $0.023 \pm 0.015$ \\
\hline $0535.0-0411$ & 053501.3 & -041154.4 & K1 & -0.10 & 0.41 & $0.038 \pm 0.014$ \\
\hline $0535.3-0059$ & 053515.2 & -005940.0 & K3 & -2.75 & 0.39 & $0.050 \pm 0.015$ \\
\hline $0535.6-0152$ & 053534.1 & -015222.8 & G9 & +2.40 & 0.32 & $0.018 \pm 0.011$ \\
\hline $0535.8-0508$ & 053545.0 & -050841.2 & K6 & -2.40 & 0.48 & $0.147 \pm 0.029$ \\
\hline $0535.7-0418$ & 053542.0 & -041808.7 & K3 & -0.15 & - & $0.050 \pm 0.015$ \\
\hline $0536.0-0650^{\dagger}$ & 053600.1 & -065009.1 & M1 & -8.57 & - & $0.056 \pm 0.020$ \\
\hline $0536.2-0519$ & 053611.8 & -051959.0 & K4 & -24.70 & 0.45 & $0.050 \pm 0.015$ \\
\hline $0536.7+0907$ & 053639.6 & +0907 16.4 & K1 & +0.60 & 0.54 & $0.049 \pm 0.014$ \\
\hline $0536.9+0608$ & 053652.8 & +06 0809.3 & K0 & +1.50 & 0.44 & $0.050 \pm 0.015$ \\
\hline $0537.0-0300$ & 053657.6 & -030039.0 & G2 & +1.85 & 0.25 & $0.127 \pm 0.025$ \\
\hline $0537.6-0054$ & 053733.7 & -005400.9 & K4 & +0.35 & 0.31 & $0.039 \pm 0.014$ \\
\hline $0538.4-0637 a$ & 053824.0 & -063747.2 & K1 & -0.15 & 0.45 & $0.060 \pm 0.018$ \\
\hline $0538.4-0637 \mathrm{~b}$ & 053824.0 & -063747.2 & $\mathrm{~K} 2$ & -0.35 & 0.42 & $0.060 \pm 0.018$ \\
\hline $0538.6-0856$ & 053834.8 & -085639.4 & G7 & +1.35 & 0.35 & $0.242 \pm 0.035$ \\
\hline $0538.8+1302$ & 053845.9 & +130257.4 & K2 & +2.67 & 0.22 & $0.032 \pm 0.011$ \\
\hline $0538.9-1321$ & 053851.3 & -132121.4 & G0 & +3.05 & 0.19 & $0.031 \pm 0.015$ \\
\hline $0538.9-0249$ & 053854.1 & -024900.5 & K3 & -0.01 & 0.40 & $0.063 \pm 0.019$ \\
\hline $0538.9-0624$ & 053856.8 & -062435.2 & G9 & +2.22 & 0.25 & $0.101 \pm 0.028$ \\
\hline $0539.2+0101$ & 053914.1 & +010101.3 & G2 & +0.20 & 0.30 & $0.076 \pm 0.017$ \\
\hline $0539.3+0918$ & 053920.7 & +09 1826.3 & K1 & +0.90 & 0.43 & $0.044 \pm 0.014$ \\
\hline $0539.4-0346$ & 053926.8 & -034654.0 & G8 & +2.78 & 0.35 & $0.060 \pm 0.015$ \\
\hline $0539.6-0242$ & 053936.1 & $\begin{array}{lll}-02 & 42 & 03.4\end{array}$ & K0 & -2.38 & 0.38 & $0.071 \pm 0.016$ \\
\hline $0539.8-0205$ & 053945.0 & $\begin{array}{lll}-02 & 05 & 00.3\end{array}$ & K4 & -4.70 & 0.53 & $0.052 \pm 0.014$ \\
\hline $0539.8-0138$ & 053948.2 & -013839.6 & K3 & -0.25 & 0.45 & $0.038 \pm 0.013$ \\
\hline $0539.9-0443$ & 053953.0 & -044325.3 & F9 & +3.15 & 0.25 & $0.052 \pm 0.013$ \\
\hline $0539.9+0915$ & 053955.4 & +09 1538.3 & K0 & +0.55 & 0.36 & $0.058 \pm 0.014$ \\
\hline $0539.9+0956$ & 053956.7 & +095640.8 & $\mathrm{~K} 4$ & -0.10 & 0.31 & $0.377 \pm 0.036$ \\
\hline $0540.1-0627$ & 054005.5 & -062749.5 & K7-M0 & -4.90 & - & $0.013 \pm 0.000$ \\
\hline $0540.1-0737$ & 054005.8 & -073759.0 & K3 & -2.64 & 0.25 & $0.050 \pm 0.015$ \\
\hline $0540.2-0708$ & 054013.8 & -070828.6 & K0 & +0.45 & 0.35 & $0.033 \pm 0.013$ \\
\hline $0540.5-0121$ & 054032.6 & -012157.0 & K5 & -0.50 & 0.40 & $0.048 \pm 0.013$ \\
\hline $0540.8-0806$ & $0540 \quad 45.9$ & -080636.1 & K5 & -0.80 & 0.58 & $0.028 \pm 0.010$ \\
\hline $0541.3+0027$ & 054118.5 & +002741.2 & $\mathrm{~K} 2$ & +0.10 & 0.46 & $0.038 \pm 0.013$ \\
\hline $0541.4-0324$ & 054123.8 & -032443.3 & K1 & +0.85 & 0.45 & $0.049 \pm 0.013$ \\
\hline $0541.8+0944$ & 054145.1 & +094435.5 & G6 & +2.70 & 0.22 & $0.044 \pm 0.014$ \\
\hline $0541.9-0556$ & 054156.1 & -055643.7 & K5 & -2.51 & 0.56 & $0.052 \pm 0.015$ \\
\hline $0542.4-0626$ & 054225.9 & -062617.6 & G7 & +1.87 & 0.30 & $0.043 \pm 0.014$ \\
\hline $0542.7-0925$ & 054239.6 & -092506.1 & K1 & +1.20 & 0.38 & $0.034 \pm 0.012$ \\
\hline $0542.9-0718^{\dagger}$ & 054256.1 & -071846.0 & M3 & -8.90 & - & $0.115 \pm 0.021$ \\
\hline
\end{tabular}

misclassifications, since the $\mathrm{H} \alpha$ emission can be strongly variable in some WTTS (Alcalá et al. 1993).

The $\mathrm{H} \alpha$ emission of most of the new PMS stars found in Orion is indeed weak, but with a few exceptions. The $\mathrm{H} \alpha$ emission line in the spectrum of RXJ0513.1+0851 is broad (FWHM $\leq 300 \mathrm{~km} \mathrm{~s}^{-1}$ ) with $W(\mathrm{H} \alpha)=-19.5$ $\pm 0.30 \AA$. The He I $\lambda 5876.7$ and $\lambda 6678.7$ lines are also present in emission (Fig. 3). The He I $\lambda 5876.7$ line is the $4^{3} \mathrm{D} \rightarrow 3^{3} \mathrm{P}$ transition, and the He I $\lambda 6678.7$ line is its singlet $4^{1} \mathrm{D} \rightarrow 3^{1} \mathrm{P}$ analogue. The ratio of the intensities of these two emission lines in the spectrum of this star is $3: 1$, which corresponds to the ratio for a dense gas in thermodynamic equilibrium. The star is relatively bright
$(V \approx 13.0)$, its photospheric spectrum is that of a K3 star and its near IR colours $(H-K=0.15$ and $J-H=0.65)$ indicate the lack of IR excess (see Fig. 5).

The optical counterparts of RXJ0523.1-0440 and RXJ0536.2-0519 show $\mathrm{H} \alpha$ in emission with $W(\mathrm{H} \alpha)=$ $-11.60 \pm 0.20 \AA$ and $W(\mathrm{H} \alpha)=-24.7 \pm 0.25 \AA$ (see Table 3 ) respectively. Unfortunately, near IR photometry for these two stars is lacking. On the basis only of their $\mathrm{H} \alpha$ emission, these three stars could be classified as CTTS. However, the forbidden lines [SII] $\lambda \lambda$ 4068, 4132, 6717 and $6731 \AA$ and [OI] $\lambda \lambda 6300$ and $6363 \AA$ are not present in their spectra, suggesting that the emission lines are of chromospheric origin. Thus, we conclude that these stars 
Table 3. continued

\begin{tabular}{|c|c|c|c|c|c|c|}
\hline$\overline{\mathrm{RXJ}}$ & $\begin{array}{c}\alpha(2000) \\
\mathrm{h} \text { m } \mathrm{s} \\
(2)\end{array}$ & $\begin{array}{c}\delta(2000) \\
\circ \quad, \quad \prime \\
(3)\end{array}$ & Sp.T. & $\begin{array}{r}W(\mathrm{H} \alpha) \\
{[\AA]} \\
(5) \\
\end{array}$ & $\begin{array}{c}W(\mathrm{Li}) \\
{[\AA]} \\
(6) \\
\end{array}$ & $\begin{array}{c}\text { cts/s } \\
(7) \\
\end{array}$ \\
\hline $0543.5-0642$ & $\begin{array}{lll}05 & 43 & 27.4\end{array}$ & -064257.0 & G9 & +1.41 & 0.19 & $0.032 \pm 0.011$ \\
\hline $0544.2-0941$ & $05 \quad 4408.3$ & -094136.9 & G9 & +2.40 & 0.30 & $0.035 \pm 0.012$ \\
\hline $0544.2-1306$ & $05 \quad 44 \quad 10.3$ & -130630.8 & K1 & +0.72 & 0.25 & $0.025 \pm 0.011$ \\
\hline $0544.2+0115$ & $\begin{array}{lll}05 & 44 & 12.4\end{array}$ & +011542.6 & K3 & +0.70 & 0.32 & $0.049 \pm 0.014$ \\
\hline $0544.6-0121$ & $05 \quad 4434.0$ & -012155.9 & K4 & -1.95 & 0.41 & $0.052 \pm 0.013$ \\
\hline $0545.6-1020$ & 054535.0 & -102026.3 & G7 & +1.85 & 0.20 & $0.054 \pm 0.015$ \\
\hline $0546.1+1232$ & $\begin{array}{lll}05 & 46 & 03.4\end{array}$ & +123236.2 & G9 & -0.30 & 0.30 & $0.023 \pm 0.012$ \\
\hline $0546.7-1223$ & 054642.5 & -122328.8 & G5 & +2.90 & 0.20 & $0.033 \pm 0.019$ \\
\hline $0546.9-0506^{\dagger}$ & $05 \quad 4651.4$ & -050657.0 & K4 & -0.20 & - & $0.027 \pm 0.013$ \\
\hline $0549.6+0232$ & $\begin{array}{lll}05 & 49 & 37.8\end{array}$ & +023259.7 & K2 & +0.95 & 0.47 & $0.030 \pm 0.012$ \\
\hline $0550.6-1249^{\dagger}$ & $05 \quad 5034.6$ & -124910.0 & K6 & -1.20 & - & $0.023 \pm 0.013$ \\
\hline $0551.2+0748^{\dagger}$ & 055109.3 & +074857.0 & K6 & +1.05 & - & $0.029 \pm 0.014$ \\
\hline $0551.2-0653$ & $\begin{array}{l}05 \\
51 \quad 10.8\end{array}$ & -065329.7 & K1 & +1.50 & 0.43 & $0.037 \pm 0.015$ \\
\hline $0552.3-0557$ & $\begin{array}{ll}05 & 5219.8\end{array}$ & -055748.9 & K2 & +1.35 & 0.33 & $0.027 \pm 0.013$ \\
\hline $0554.6-0736$ & $05 \quad 5434.1$ & -073629.3 & G6 & +2.68 & 0.22 & $0.025 \pm 0.013$ \\
\hline $0556.1-0800$ & 055608.0 & $\begin{array}{llll}-08 & 00 & 17.5\end{array}$ & G8 & +2.09 & 0.30 & $0.046 \pm 0.017$ \\
\hline $0556.5+0619$ & $\begin{array}{lll}05 & 56 & 28.1\end{array}$ & +06 1937.3 & G9 & +2.79 & 0.19 & $0.033 \pm 0.015$ \\
\hline $0556.8-0611^{\dagger}$ & 055646.8 & -061113.5 & K5 & -0.15 & - & $0.038 \pm 0.015$ \\
\hline $0558.0+0929$ & 055757.5 & +092903.4 & G9 & +3.50 & 0.27 & $0.025 \pm 0.013$ \\
\hline $0558.0-0145$ & $\begin{array}{llll}05 & 58 & 02.1\end{array}$ & -014529.5 & K1 & +2.05 & 0.27 & $0.036 \pm 0.016$ \\
\hline
\end{tabular}

${ }^{\dagger}$ Li I $\lambda 6707 \AA$ absorption to be confirmed because of the low signal-to-noise of the spectrum.

$\ddagger$ optical counterpart of the source RXJ 0534.7-0524. The X-ray position of the the RASS source is given.

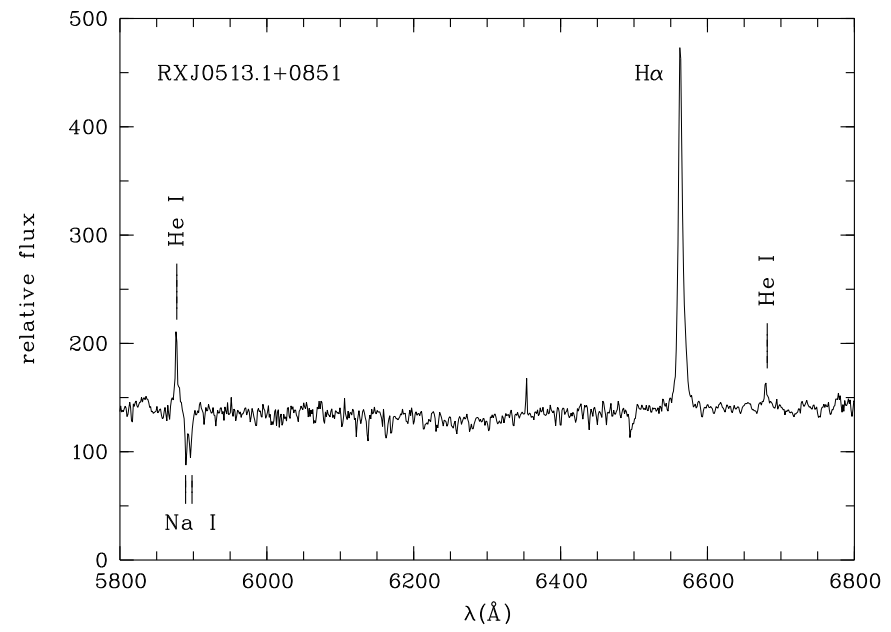

Fig. 3. Spectrum of the optical counterpart of the source RXJ0513.1+0851

are not CTTS, but most probably WTTS with very active chromospheres or WTTS which presented flare activity during the spectroscopic observations. The latter hypothesis is supported by studies of flare activity in WTTS in Orion (Preibish et al. 1995, and references therein) and the recent finding that some of the RASS discovered WTTS in the Chamaeleon SFR show flare activity too (Guenther 1995). A few of these stars, while displaying normally a typical WTTS spectrum, sometimes show a spectrum with characteristics very similar to those of the spectrum in Fig. 3 (Alcalá et al. 1993; Alcalá et al., in preparation).

\subsection{Spatial distribution of the new WTTS}

The spatial distribution of the new WTTS and the known CTTS from the Herbig \& Bell catalogue (1988) in the Orion cloud complex is shown in Fig. 4. The outlines of the CO maps by Maddalena et al. (1986) are also overplotted for comparison purposes. While the WTTS are more or less uniformly spread over the whole star forming region, the CTTS are concentrated in the denser parts or cores of the molecular clouds. This confirms previous results found for the Chamaeleon and Taurus-Auriga SFRs (A95; W95).

Most of the CTTS in the Orion complex are located in the Orion nebula, the NGC 2023 and NGC 2024 clusters and in the $\lambda$ Orionis region. Many WTTS are located also in these areas, but plenty of them are also scattered southwards and northwards of the nebula, where no known CTTS are found and they extend even farther off the limits of the "A" molecular cloud. There are also WTTS located near the NGC 2023 and NGC 2024 clusters and the $\lambda$ Orionis region, but they are much more scattered than the CTTS. Note that some WTTS are more than $6^{\circ}$ distant from the cloud cores. Assuming typical T Tauri ages of $\leq 10^{7}$, the outermost WTTS should move with mean velocities greater than the typical space velocity given by the velocity dispersion of the CTTS. The existence of lowmass PMS stars rather far from SFRs has been interpreted in terms of "run-away T Tauri stars" (RATTS) by Sterzik et al. (1995), who used 98 of the 112 new WTTS as training sample to preselect potential PMS candidates. The rest of the new WTTS were identified later but independently form the Sterzik et al. (1995) criterion. We investigated the statistical status of these $14 \mathrm{X}$-ray sources in the 
Table 4. Journal of photometric observations

\begin{tabular}{lllll}
\hline Period & Telescope & Detector & $\begin{array}{l}\text { Photometric } \\
\text { system }\end{array}$ & $\begin{array}{l}\text { No. of target } \\
\text { stars }\end{array}$ \\
\hline November 1991 & $0.8 \mathrm{~m}$ & P07029 & $U B V(R I)_{\mathrm{KC}}$ & 6 \\
November 1992 & $2.1 \mathrm{~m}$ & InSb & $J H K L M$ & 45 \\
November 1992 & $0.8 \mathrm{~m}$ & P07029 & $U B V(R I)_{\mathrm{KC}}$ & 10 \\
December 1992 & $1.5 \mathrm{~m}$ & P07029 & $U B V(R I)_{\mathrm{KC}}$ & 8 \\
January 1994 & $1.5 \mathrm{~m}$ & EMI9789QA & $u v b y-\beta$ & 20 \\
November 1994 & $1.5 \mathrm{~m}$ & EMI9789QA & $u v b y-\beta$ & 23 \\
December 1994 & $0.8 \mathrm{~m}$ & P07029 & $U B V(R I)_{\mathrm{KC}}$ & 15 \\
\hline
\end{tabular}

context of the pre-selection criterion and found that most of them can be classified as potential $\mathrm{T}$ Tauri candidates. We have also checked the status the sources with dubious lithium absorption and obtained similar results. A more complete study of the relation between spatial distribution of the WTTS and their ages will be published in our forthcoming paper.

\subsection{Finding charts of the new WTTS in Orion}

Finding charts for the new WTTS (excluding RXJ 0534.70524 which is identified with the star HBC447, see Sects. 3.1 and 3.2) were produced using the Palomar digitised photographic plates and are provided in Fig. 6. In these finding charts North is up, and East is to the left. Each finding chart covers a field of $5^{\prime} \times 5^{\prime}$ on the sky. The finding charts are sorted by right ascension, and the name of the corresponding X-ray source is also reported for identification in Table 3.

\section{The photometric observations}

$U B V(R I)_{\mathrm{KC}}, J H K L$ and $u v b y-\beta$ photometric observations for 39, 45 and 42 new WTTS respectively, were carried out at the San Pedro Mártir National Astronomical Observatory (SPMO) in Baja California, México. The data were gathered during several observational runs in the period from November 1991 to December 1994. The basic information on the observing runs, the telescopes and the instrumental set-up used are reported in Table 4 .

\section{1. $U B V(R I)_{\mathrm{KC}}$ photometry}

The $84 \mathrm{~cm}$ telescope and the Lowell I pulse counting photometer of the Sierra San Pedro Mártir National Astronomical Observatory in Baja California, Mexico (SPMO) were used to acquire the $U B V(R I)_{\mathrm{KC}}$ photometry of the new Orion WTTS detected with ROSAT. The photometer consists of a P07029 photomultiplier in the pulse counting mode cooled to $-70^{\circ} \mathrm{C}$ and a set of Johnson-Kron-Cousins $\left(U B V(R I)_{\mathrm{KC}}\right)$ filters. Details about the instrument can be found in a technical report by Echevarria et al. (1991). A set of 15 reference stars were observed nightly in order to tie the observations to the $U B V(R I)_{\mathrm{KC}}$ standard system. The reference stars were selected from the list by

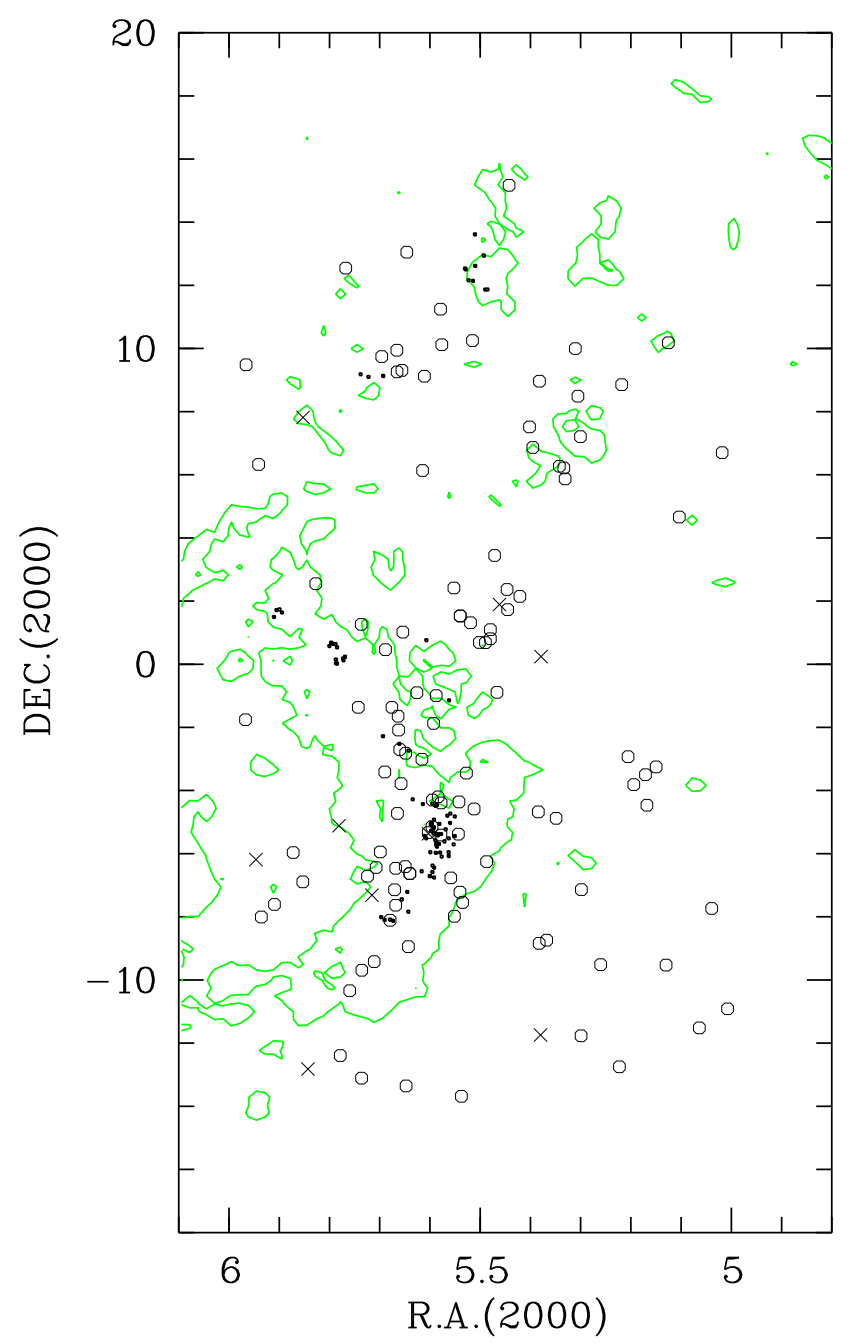

Fig. 4. Spatial distribution of the newly discovered WTTS (o) and of the known CTTS (.) in the Orion SFR. The crosses $(\times)$ represent WTTS candidates for which the Li I $\lambda$ $6707 \AA$ absorption line has to be confirmed. The outlines of the CO line radio-survey by Maddalena et al. (1986) are overplotted as a shaded line. See Sect. 3.5 for further explanations 
Landolt (1983) and span from B7 to M0 spectral types. The observations consisted of a of several (10-15 s) integrations on each filter on the star and on the near background. A 20 arcsec diaphragm was used. The data were reduced following the procedures by outlined by Harris et al. (1981). Seasonal mean extinction coefficients given by Schuster (1982) were adopted for the atmospheric extinction correction. The resulting $U B V(R I)_{\mathrm{KC}}$ photometry of the program stars is presented in Table 5 . The typical observing errors were estimated from the zero point scatter of the standard stars and are the following: $\sigma_{U}=0.05$, $\sigma_{B}=0.04, \sigma_{V}=0.03, \sigma_{R_{\mathrm{KC}}}=0.02$ and $\sigma_{I_{\mathrm{KC}}}=0.02$.

\subsection{JHKL photometry}

The SPMO near-IR photometer consists of a InSb diode as detector (cooled at the liquid $N_{2}$ temperature) and a $J H K L M$ set of filters. The photometer was attached to the $2.1 \mathrm{~m}$ telescope in its infrared configuration $(f / 27)$ with a chopping secondary mirror (see Roth et al. 1984 for details). A 40 arcsec throw in declination axis and a 10 arcsec diaphragm were used during the observations. In order to fix the observations to the local photometric system, we observed $5 \pm 1$ near IR standard stars nightly. These stars were taken from the list by Tapia et al.(1986). The data at each night were reduced independently following a standard procedure (e.g. Mitchell 1960). The resulting photometry of the program stars is given in Table 5. Typical errors of the derived magnitudes of the program stars were estimated from integrations in each filter and from the nightly zero point dispersion of the standard stars. The mean errors are: $\sigma_{J}=0.03, \sigma_{H}=0.03, \sigma_{K}=0.05$ and $\sigma_{L}=0.30$.

The IR colour-colour diagram for the newly discovered WTTS in Orion is shown in Fig. 5 together with the sample of class I and class II IR-sources in the Taurus-Auriga SFR taken from the literature (Herbig \& Bell 1988). The normal reddening vector is also shown. Except for three stars (RXJ0534.7-0423, RXJ0535.0-0411 and RXJ0539.80205), it can be seen that the WTTS tend to follow the line given by the intrinsic colour line for normal field dwarfs, indicating the lack of (significant) near IR flux-excesses. In the two-colour diagram of Fig. 5, three groups are clearly distinguished: the first group is represented by the IRsources, the WTTS form the second group and the third group is defined by the field stars. The apparent difference between WTTS and field stars in the IR colour-colour diagram can be ascribed to their different spectral type distributions. While most WTTS have spectral types later than G4, most field stars are earlier than G5.

The spectra of the three discrepant WTTS in the IR colour-colour diagram do not show any untypical characteristic, but we cannot exclude the possible presence of residual circumstellar matter in these stars. For a subsample of 16 WTTS with both $U B V(R I)_{\mathrm{KC}}$ and $J H K$ pho- tometric measurements, we derived the spectral energy distributions (SEDs) and confirmed that the new WTTS are "class III" IR sources.

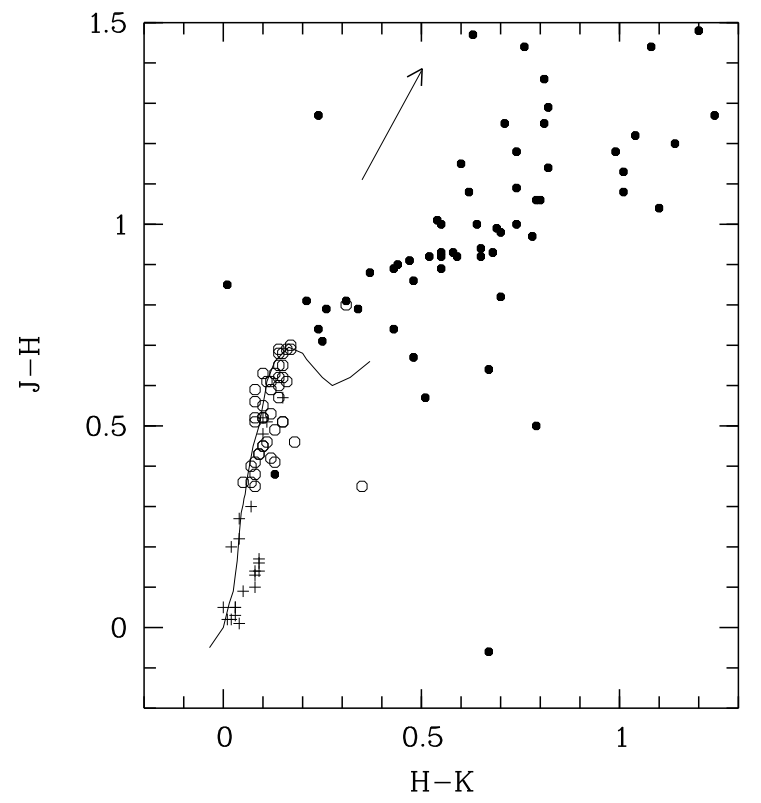

Fig. 5. IR colour-colour diagram for three groups of stars: the new WTTS in Orion (open circles), the class I and class II IR-sources in Taurus-Auriga (filled circles) and normal field stars (crosses). The solid line represents the intrinsic colours for dwarfs from Bessel \& Brett (1988) and arrow indicates the normal reddening vector

\section{3. uvby- $\beta$ photometry}

The SPMO "Danish" grating spectrophotometer for the $u v b y-\beta$ photometry is described in detail by Nissen (1984) and by Schuster \& Nissen (1988). For a brief description of the photometer consult Terranegra et al. (1994). The photometer consists of six rectangular slots, their corresponding "spectral edge smoothing" filters and sic photomultipliers in a photon counting mode. Four channels are used for simultaneous uvby observations and the two remaining channels (the "wide" and "narrow" passbands) are used for the $\beta$-index measurements. In both modes, the channels are measured simultaneously but independently from another. Uncooled EMI 9789 QA type photomultipliers are used as detectors in the photon counting mode. The system closely reproduces the uvby- $\beta$ systems of Crawford \& Barnes (1970) and of Olsen (1983, 1984). About 15 secondary standard stars were observed from a list kindly provided to us by W. Schuster. Care was taken to include standard stars covering the spectral types and luminosity classes of the program stars. A 20 arcsec diaphragm was used during the observations. The integration time was fixed in order to achieve a photon noise less than $1 \%$ for the net signal. All the standard stars were observed at 
Table 5. $U B V(R I)_{\mathrm{KC}}$ and near IR photometry of the new WTTS in Orion

\begin{tabular}{|c|c|c|c|c|c|c|c|c|c|}
\hline$\overline{\mathrm{RXJ}}$ & $U$ & $B$ & $V$ & $R_{\mathrm{KC}}$ & $I_{\mathrm{KC}}$ & $J$ & $H$ & $K$ & $L$ \\
\hline$\overline{0500.4-1054}$ & & 14.11 & 13.00 & 12.31 & 11.58 & & & & \\
\hline $0510.3-0330$ & & 12.53 & 11.74 & 11.29 & 10.87 & & & & \\
\hline $0513.1+0851$ & & & & & & 10.20 & 9.55 & 9.40 & \\
\hline $0515.6-0930$ & & 10.46 & 9.79 & 9.39 & +9.02 & & & & \\
\hline $0518.0+0712$ & & & & & & 10.40 & 9.89 & 9.74 & \\
\hline $0518.3+0829$ & 12.54 & 12.04 & 11.15 & 10.54 & 10.11 & 9.40 & 8.91 & 8.78 & \\
\hline $0520.0+0612$ & 13.46 & 12.50 & 11.31 & 10.60 & 10.09 & 9.17 & 8.58 & 8.46 & 8.62 \\
\hline $0520.5+0616$ & 13.88 & 12.68 & 11.49 & 10.80 & 10.14 & 9.18 & 8.61 & 8.47 & 8.41 \\
\hline $0520.9-0452$ & & 9.98 & 9.53 & 9.24 & 8.97 & & & & \\
\hline $0522.1-0844$ & 13.59 & 12.95 & 12.09 & 11.60 & 11.13 & & & & \\
\hline $0522.9+0857$ & & 11.27 & 10.28 & & 9.08 & 8.12 & 7.52 & 7.38 & 7.30 \\
\hline $0523.7+0652$ & 14.18 & 13.87 & 12.47 & 11.37 & 10.83 & 9.83 & 9.15 & 9.00 & 8.78 \\
\hline $0526.7+0143$ & & & & & & 10.01 & 9.66 & 9.58 & 8.84 \\
\hline $0526.8+0222$ & 12.29 & 12.18 & 11.51 & 11.11 & 10.71 & 10.05 & 9.69 & 9.62 & 7.42 \\
\hline $0528.0-0053$ & & & & & & 10.74 & 10.23 & 10.15 & \\
\hline $0528.3+0326$ & 13.09 & 12.66 & 11.76 & 11.18 & 10.81 & 9.97 & 9.45 & 9.35 & 8.89 \\
\hline $0528.8+0048$ & & & & & & 11.31 & 10.90 & 10.77 & \\
\hline $0528.8+0105$ & & & & & & 10.19 & 9.51 & 9.37 & 8.79 \\
\hline $0529.2-0615$ & 12.42 & 12.12 & 11.27 & 10.77 & 10.31 & & & & \\
\hline $0529.4+0041$ & & & & & & 10.32 & 9.69 & 9.59 & \\
\hline $0530.1+0041$ & 10.33 & 10.23 & 9.54 & 9.19 & 8.72 & 8.15 & 7.74 & 7.66 & 8.00 \\
\hline $0530.7-0434$ & 13.90 & 12.57 & 11.51 & 10.79 & 10.25 & 9.28 & 8.60 & 8.45 & 7.90 \\
\hline $0531.2+0118$ & & 13.68 & 12.84 & 12.36 & 11.88 & & & & \\
\hline $0532.1-0732$ & & & & & & 10.55 & 9.94 & 9.83 & \\
\hline $0532.2-1340$ & & 10.21 & 9.67 & 9.32 & 9.07 & 8.63 & 8.43 & 8.45 & 7.99 \\
\hline $0532.4-0713$ & 14.03 & 13.97 & 12.86 & 12.23 & 11.57 & 10.70 & 10.05 & 9.91 & \\
\hline $0532.5-0421$ & 13.80 & 13.05 & 11.93 & 11.27 & 10.65 & & & & \\
\hline $0532.6-0522$ & 14.62 & 13.19 & 12.05 & 11.32 & 10.37 & 9.72 & 9.03 & 8.87 & \\
\hline $0533.1-0758$ & & & & & & 9.87 & 9.25 & 9.10 & 9.93 \\
\hline $0533.5-0646$ & 12.11 & 11.85 & 11.12 & 10.70 & 10.28 & & & & \\
\hline $0534.7-0423$ & 12.55 & 11.53 & 11.06 & 10.73 & 9.92 & 10.12 & 9.86 & 9.80 & \\
\hline $\mathrm{HBC} 447$ & 13.88 & 13.61 & 12.34 & 11.80 & 11.25 & & & & \\
\hline $0535.0-0411$ & & & & & & 10.27 & 9.92 & 9.57 & \\
\hline $0535.3-0059$ & & 15.10 & 14.06 & 13.22 & 12.61 & & & & \\
\hline $0535.8-0508$ & 15.61 & 14.41 & 12.91 & 12.09 & 11.31 & & & & \\
\hline $0536.7+0907$ & & & & & & 10.43 & 9.82 & 9.70 & \\
\hline $0536.9+0608$ & & & & & & 10.81 & 10.36 & 10.26 & \\
\hline $0537.0-0300$ & 11.11 & 10.97 & 10.25 & 9.79 & 9.37 & 8.91 & 8.48 & 8.39 & 8.59 \\
\hline $0537.6-0054$ & & & & & & 9.67 & 9.04 & 8.91 & \\
\hline $0538.6-0856$ & & & & & & 8.50 & 8.04 & 7.93 & 7.69 \\
\hline $0538.9-1321$ & & 11.20 & 10.63 & 10.29 & 9.96 & & & & \\
\hline $0538.9-0249$ & & & & & & 10.93 & 10.32 & 10.16 & \\
\hline $0538.9-0624$ & & & & & & 9.63 & 9.23 & 9.16 & 8.55 \\
\hline $0539.2+0101$ & & & & & & 9.73 & 9.20 & 9.08 & 8.68 \\
\hline $0539.4-0346$ & & & & & & 9.78 & 9.33 & 9.23 & \\
\hline $0539.6-0242$ & 11.21 & 10.92 & 10.06 & 9.51 & 9.11 & 8.53 & 8.01 & 7.91 & 7.78 \\
\hline $0539.8-0205$ & & & & & & 10.75 & 9.95 & 9.64 & \\
\hline $0539.9-0443$ & & & & & & 9.61 & 9.25 & 9.20 & 10.00 \\
\hline $0540.1-0737$ & & & & & & 9.66 & 9.20 & 9.02 & \\
\hline $0540.2-0708$ & & & & & & 10.81 & 10.29 & 10.21 & \\
\hline $0540.8-0806$ & & 14.23 & 12.87 & 12.10 & 11.29 & & & & \\
\hline $0541.3+0027$ & & & & & & 10.95 & 10.26 & 10.09 & \\
\hline $0541.4-0324$ & 12.63 & 12.70 & 11.75 & 11.18 & 10.66 & 10.07 & 9.51 & 9.43 & \\
\hline $0541.8+0944$ & 10.88 & 10.73 & 10.07 & 9.67 & 9.28 & & & & \\
\hline $0541.9-0556$ & & & & & & 10.74 & 10.02 & 9.96 & \\
\hline $0542.4-0626$ & & & & & & 10.52 & 10.14 & 10.06 & 9.80 \\
\hline $0542.7-0925$ & & & & & & 10.51 & 9.96 & 9.86 & \\
\hline $0543.5-0642$ & & & & & & 10.62 & 10.19 & 10.10 & 9.42 \\
\hline $0544.2-0941$ & 13.21 & 12.93 & 12.22 & 11.77 & 11.35 & & & & \\
\hline $0544.2-1306$ & & & & & & 9.11 & 8.49 & 8.35 & 7.76 \\
\hline $0544.2+0115$ & & & & & & 10.01 & 9.42 & 9.34 & \\
\hline $0545.6-1020$ & 12.33 & 12.10 & 11.43 & 11.01 & 10.61 & & & & \\
\hline $0549.6+0232$ & 13.86 & 13.24 & 12.33 & 11.75 & 11.20 & & & & \\
\hline $0551.2-0653$ & 12.51 & 12.08 & 11.24 & 10.73 & 10.26 & & & & \\
\hline $0554.6-0736$ & 12.43 & 12.33 & 11.71 & 11.31 & 10.93 & & & & \\
\hline $0556.1-0800$ & 13.11 & 12.78 & 12.04 & 11.59 & 11.19 & & & & \\
\hline $0556.5+0619$ & 11.53 & 11.19 & 10.41 & 9.96 & 9.52 & & & & \\
\hline $0558.0-0145$ & 13.55 & 12.93 & 12.08 & 11.55 & 11.08 & & & & \\
\hline
\end{tabular}


least twice during the run. The instrumental magnitudes and colors were transformed to the standard system following usual procedures (cf. Terranegra et al. 1994). The NABAPHOT utility package of the San Pedro Mártir Observatory was used to reduce the data (Arellano-Ferro \& Parrao 1988). The resulting uvby- $\beta$ photometry of the program stars observed is listed in Table 6 . In this table we give the quantities $c_{1}, m_{1}$ (cf. Strömgren 1966) and the $\beta$ index (Crawford 1979). The typical mean errors of these measured quantities are $\sigma_{y}=0.20, \sigma_{b-y}=0.05, \sigma_{c_{1}}=0.08$, $\sigma_{m_{1}}=0.05$ and $\sigma_{H \beta}=0.06$.

Table 6. $u v b y-\beta$ photometry

\begin{tabular}{crccll}
\hline RXJ & $V$ & $b-y$ & $m_{1}$ & $c_{1}$ & $H \beta$ \\
\hline $0500.4-1054$ & 13.03 & 0.67 & 0.43 & 0.27 & $2.46^{e, v ?}$ \\
$0501.1+0642$ & 12.62 & 0.64 & 0.44 & - & 2.55 \\
$0502.4-0744$ & 11.21 & 0.46 & 0.20 & 0.38 & 2.58 \\
$0506.2+0439$ & 13.58 & 1.15 & - & 0.30 & $2.37^{e}$ \\
$0507.5+1010$ & 10.45 & 0.47 & 0.24 & 0.27 & 2.60 \\
$0509.0-0315$ & 11.27 & 0.48 & 0.21 & 0.32 & 2.57 \\
$0510.1-0427$ & 11.58 & 0.61 & 0.51 & 0.12 & 2.53 \\
$0510.3-0330$ & 11.38 & 0.52 & 0.22 & 0.32 & 2.57 \\
$0511.7-0348$ & 11.93 & 0.51 & 0.33 & 0.23 & 2.59 \\
$0512.3-0255$ & 10.47 & 0.46 & - & 0.20 & $2.43^{e}$ \\
$0513.1+0851$ & 13.08 & 0.80 & 0.37 & 0.26 & 2.60 \\
$0513.4-1244$ & 10.77 & 0.38 & 0.21 & 0.34 & 2.61 \\
$0515.6-0930$ & 9.84 & 0.42 & 0.27 & 0.28 & 2.59 \\
$0517.9-0708$ & 10.70 & 0.52 & 0.35 & 0.27 & 2.56 \\
$0518.0-1146$ & 11.51 & 0.55 & 0.36 & 0.25 & 2.58 \\
$0518.0+0712$ & 13.08 & 0.63 & 0.32 & 0.36 & 2.63 \\
$0518.3+0829$ & 11.68 & 0.54 & 0.25 & 0.35 & 2.58 \\
$0520.0+0612$ & 11.56 & 0.70 & 0.47 & 0.27 & 2.55 \\
$0520.5+0616$ & 12.20 & 0.69 & 0.43 & 0.28 & 2.56 \\
$0520.9-0452$ & 9.48 & 0.31 & 0.16 & 0.40 & 2.64 \\
$0522.9+0857$ & 11.13 & 0.61 & 0.21 & 0.36 & 2.59 \\
$0523.7+0652$ & 12.79 & 0.84 & 0.45 & 0.23 & 2.60 \\
$0526.7+0143$ & 12.82 & 0.62 & 0.25 & 0.50 & 2.45 \\
$0526.8+0222$ & 11.75 & 0.46 & 0.18 & 0.43 & 2.62 \\
$0528.3+0326$ & 12.04 & 0.59 & 0.27 & 0.37 & 2.60 \\
$0528.8+0048$ & 14.04 & 0.59 & 0.23 & 0.33 & 2.62 \\
$0529.2-0615$ & 11.19 & 0.53 & 0.26 & 0.27 & 2.54 \\
$0529.4+0041$ & 12.56 & 0.64 & 0.41 & 0.28 & 2.55 \\
$0530.1+0041$ & 9.53 & 0.44 & 0.19 & 0.39 & 2.63 \\
$0530.7-0434$ & 11.57 & 0.72 & 0.41 & 0.27 & 2.59 \\
$0532.1-0732$ & 12.57 & 0.64 & 0.37 & 0.34 & 2.55 \\
$0532.2-1340$ & 9.61 & 0.35 & 0.17 & 0.41 & 2.54 \\
$0532.4-0713$ & 12.82 & 0.67 & 0.32 & 0.33 & 2.62 \\
$0532.5-0421$ & 11.89 & 0.63 & 0.46 & 0.24 & 2.58 \\
$0532.6-0522$ & 12.19 & 0.73 & 0.43 & 0.26 & 2.57 \\
$0533.1-0758$ & 11.76 & 0.71 & 0.43 & 0.35 & 2.56 \\
$0534.7-0423$ & 13.22 & 0.68 & 0.43 & 0.32 & 2.66 \\
$0535.0-0411$ & 12.02 & 0.58 & 0.28 & 0.33 & 2.49 \\
$0535.3-0059$ & 12.79 & 0.73 & 0.38 & 0.44 & 2.69 \\
$0537.0-0300$ & 10.14 & 0.47 & 0.17 & 0.46 & 2.60 \\
$0538.6-0856$ & 9.92 & 0.48 & 0.25 & 0.35 & 2.59 \\
$0541.8+0944$ & 10.09 & 0.42 & 0.20 & 0.35 & 2.51 \\
& & & & & \\
\hline & & & & &
\end{tabular}

$e: H \beta$ in emission; $v$ ?: possibly variable $\mathrm{H} \beta$.

\section{Conclusions}

X-ray data from the RASS in an area of about 450 square degrees in direction to the Orion cloud complex were obtained. The mean spatially projected density of X-ray sources in this region is a factor of about two higher than that for the RASS elsewhere $5.6 \%$ of the RASS sources in this region are identified with previously known pre-main sequence stars. X-ray light curves were obtained for more than 200 sources detected with a high $S / N$ ratio. Only three sources were found to show significant variability in $\mathrm{X}$-rays, but we cannot exclude the possibility of low-level $\mathrm{X}$-ray variability, observable as scan-to-scan variations, in the rest of the sources.

181 (spatially unbiased) RASS sources, widely distributed over the entire cloud complex, were investigated spectroscopically. $62 \%$ of them could be identified with WTTS. The new WTTS are found to be widely distributed throughout the entire Orion star forming region, while the CTTS in this region tend to be concentrated mainly in the cloud cores.

A sample of 39 WTTS was observed in the $U B V(R I)_{\mathrm{KC}}$ photometric system. Near-IR $J H K L$ photometry for 45 WTTS was also obtained. In addition, $u v b y$ - $\beta$ photometric measurements were obtained for a sample of 42 WTTS. The spectral energy distributions of these WTTS and their position in the IR colour-colour diagram, confirms that they are "class III" IR sources.

Acknowledgements. We are very grateful to Prof. I. Appenzeller, E. Covino, M. Sterzik and R. Neuhäuser for valuable comments and suggestions for this research work. The ROSAT team, and in particular the EXSAS group at MPE are also acknowledged for assistance and help with the ROSAT data reduction. G. García of the SPMO efficiently assisted us with the near IR observations at the telescope and W. Schuster and L. Parrao helped us to configurate a subset of standard stars and instructing us with the reductions of the $u v b y$-beta observations, respectively The ROSAT project has been supported by the Bundesministerium für Bildung, Wissenschaft, Forschung und Technologie and the Max-Planck-Gesellschaft. This research has made use of the SIMBAD database, operated at CDS, Strasbourg, France.

\section{References}

Alcalá J.M., Covino E., Franchini M., et al., 1993, A\&A 272, 225

Alcalá J.M., Krautter J., Schmitt J.H.M.M., et al., 1995, A\&AS 114, 109 (A95)

Arellano-Ferro A., Parrao L., 1988, Techn. Report No. 57, Instituto de Astronomia, UNAM, Ap. Postal 70-264, Mexico 04510, D.F.

Blaauw A., 1991, in "The physics of star formation and early stellar evolution". In: Lada C.J., Kylafis N.D. (eds.), Vol. 342 of NATO ASI Series C. Dordrech: Kluwer

Bodenheimer P., 1965, ApJ 142, 459

Brand J., Wouterloot J.G.A., 1991, in "Low mass star formation in southern molecular clouds". In: Bo Reipurth (ed.), ESO Scientific Report No. 11, p. 1

Crawford D.L., 1979, in "Problems of Calibrations of Multicolor Photometric Systems". In: Philip A.E. (ed.), Dudley Obs. Rep. No. 14

Crawford D.L., Barnes J.V., 1970, AJ 75, 978

Duerr R, Imhoff C.L., Lada C.J., 1982, ApJ 261, 135

Echevarría J., Murillo J., Hiriart D., 1991, Manual de Usuario del Fotómetro Cuenta PulsosII, Observatorio Astronómico 
Nacional, Apartado postal 877, Ensenada 22800, B.C. México

Feigelson E.D., 1987 in Protostars and molecular clouds. In: Montmerle Th. and Bertout C. (eds.), CEN Saclay, Gif-sur-Yvette, p. 123

Gagné M., Caillault J-P., 1994, ApJ 437, 361

Genzel R., Stutzki J., 1989, ARA\&A 27, 41

Giacconi R., Gursky H., 1974, "X-ray Astronomy". Dordrecht: Reidel

Guenther E., 1995, in Proceedings of the ESO workshop "The role of dust in the formation of stars". In: Ralf Siebenmorgen and Hans Ulrich Käufl. (eds.)

Harris W.E., Fitzgerald M.P., Reed B.C., 1981, PASP 93, 507

Herbig G.H., Bell K.R., 1988, Lick Observatory Bulletin, No. 1111

Kogure T., et al., 1989, PASJ 41, 1195

Krautter J., Alcalá J.M., Wichmann R., Neuhäuser R., Schmitt J.H.M.M., 1994, Rev. Mex. Astron. Astrofis. 29, 41

Ku W.H.-M., Chanan G.A., 1979, ApJ 228, L33

Landolt A.U., 1983, AJ 88, 439

Maddalena R.J., Morris M, Moscowitz J., Thaddeuss P., 1986, ApJ 303, 375

Mitchell R., 1960, ApJ 132, 68

Neuhäuser R., Sterzik M.F., Schmitt J.H.M.M., Wichmann R., Krautter J., 1995, A\&A 297, 391

Nissen P., 1984 Technical Report of the SPMO, Instituto de Astronomía, P.O. Box 439027, San Diego, CA 92143-9027, U.S.A.
Olsen E.H., 1983, A\&AS 54, 55

Olsen E.H., 1984, A\&AS 57, 443

Pfeffermann E., et al., 1986, Proc. SPIE 733, 519

Preibish Th., Neuhäuser R., Alcalá J.M., 1995, A\&A 304, L13

Roth M, Iriarte A., Tapia M., Reséndiz G., 1984, Rev. Mex. Astron. Astrofis. 9, 25

Schuster W.J., 1982, Rev. Mex. Astron. Astrof. 5, 149

Schuster W., Nissen P., 1988, A\&AS 73, 225

Sterzik M., Alcalá J.M., Neuhäuser R., Schmitt J.H.M.M., 1995, A\&A 297, 418

Strömgren B., 1966, ARA\&A 4, 433

Tapia M., Neri L., Roth M., 1986, Rev. Mex. Astron. Astrofis. 13,115

Terranegra L., Chavarría-K. C., Díaz S., González-Patiño D., 1994, A\&AS 104, 557

Trümper J., et al., 1991, Nat 349, 579

Voges W., 1992a, Satellite Symp. No. 3 ESA ISY-3, p. 155

Voges W., 1992b, The ROSAT all-sky survey and the first identifications of X-ray sources using digitised optical plates. In: MacGillivray H.T., Thomson E.B. (eds.), Digitised optical sky surveys. Kluwer, p. 452-463

Walter F.M., Brown A., Mathieu R.D., Myers P.C., Vrba F.J., 1988, AJ 96, 297

Wichmann R., et al., 1995, A\&A (submitted) (W95)

Wiramihardja S.D., et al., 1989, PASJ 41, 155

Zimmermann, et al., 1994, EXSAS user's Guide MPE Report No. 257 
Fig. 6. Finding charts for the new WTTS. In these finding charts North is up, and East is to the left. Each finding chart covers a field of $5^{\prime} \times 5^{\prime}$ on the sky. The finding charts are sorted by right ascension, and the name of the corresponding X-ray source is at the top of each field for identification in Table 3 
Fig. 6. continued 
Fig. 6. continued 
Fig. 6. continued 